Cite as: Stephane H Maes, (2021), "The Multi-fold Theory: A synopsis so far", viXra:2105.0013v1, https://vixra.org/ pdf/2105.0013v1.pdf, https://shmaesphysics.wordpress.com/2021/05/03/the-multi-fold-theory-a-synopsis-so-far/, May 3, 2021.

\title{
The Multi-fold Theory: A synopsis so far
}

\author{
Stephane H. Maes ${ }^{1}$
}

May 3, 2021

\begin{abstract}
:
This paper provides a synopsis and review of the multi-fold theory in preparation for more detailed reviews lectures and books.

In a multi-fold universe, gravity emerges from Entanglement through the multi-fold mechanisms. As a result, gravity-like effects appear in between entangled particles that they be real or virtual. Long range, massless gravity results from entanglement of massless virtual particles. Entanglement of massive virtual particles leads to massive gravity contributions at very smalls scales. Multi-folds mechanisms also result into a spacetime that is discrete, with a random walk fractal structure and non-commutative geometry that is Lorentz invariant and where spacetime nodes and particles can be modeled with microscopic black holes. All these recover General Relativity (GR) at large scales and semi-classical model remain valid till smaller scale than usually expected. Gravity can therefore be added to the Standard Model resulting into what we defined as $S M_{G}$. This can contribute to resolving several open issues with the Standard Model without new Physics other than gravity. These considerations hints at a even stronger relationship between gravity and the Standard Model.
\end{abstract}

Among the multi-fold $S M_{G}$ discoveries, the apparition of an-always in-flight, and hence non-interacting, righthanded neutrinos, coupled to the Higgs boson is quite notable. It is supposedly always around right-handed neutrinos, due to chirality flips by gravity of the massless Weyl fermions, induced by $7 D$ space time matter models and hidden behind the Higgs boson or field at the entry points and exit points of the multi-folds. Massless Higgs bosons modeled as minimal microscopic black holes mark concretized spacetime location. They can condensate into Dirac Kerr-Newman soliton Qballs to produce massive and charged particles, thereby providing a microscopic explanation for a Higgs driven inflation, the electroweak symmetry breaking, the Higgs mechanism, the mass acquisition and the chirality of fermions and spacetime; all resulting from the multi-fold gravity electroweak symmetry breaking.

The multi-fold theory has also concrete implications on New Physics.

We see this paper as a draft to collect comment and feedback as we evolve it towards a fuller publication.

\section{Contents}

1. Introduction 3

2. Overview of Multi-fold theory

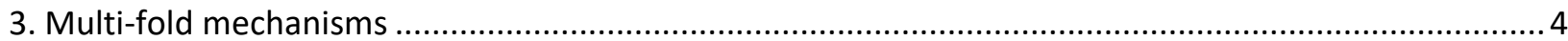

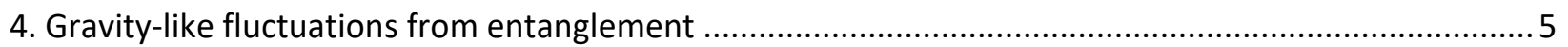

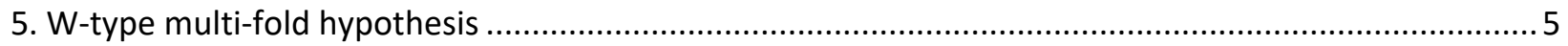

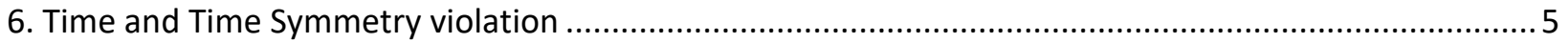

${ }^{1}$ shmaes.physics@gmail.com 
7. Gravity emergence from entanglement and the E/G Conjecture ................................................ 6

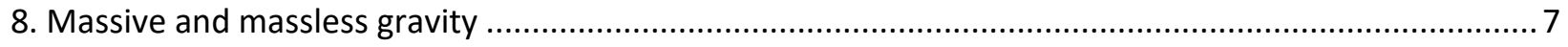

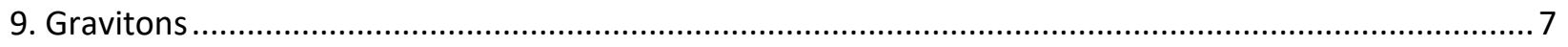

10. AdS/CFT correspondence conjecture and facts in Multi-fold universes............................................ 7

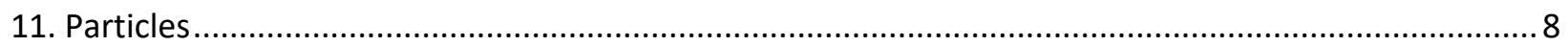

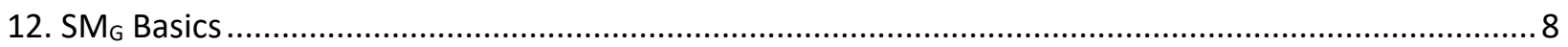

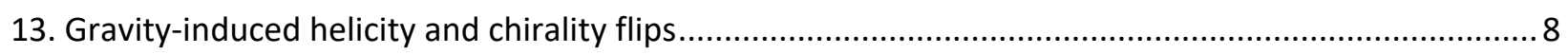

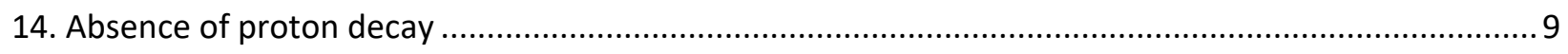

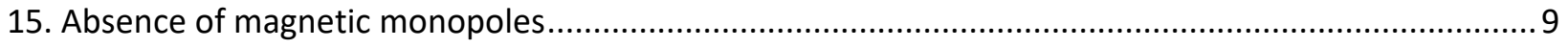

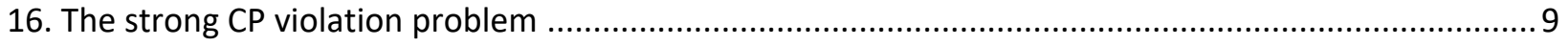

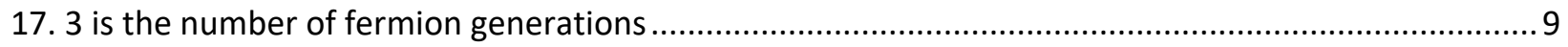

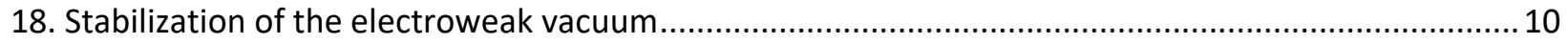

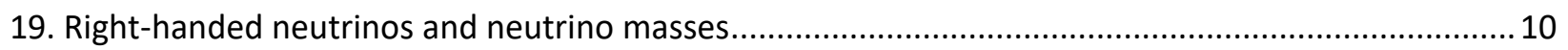

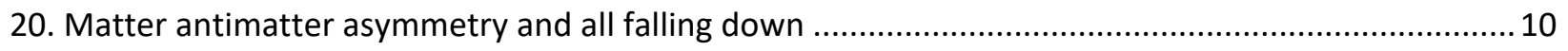

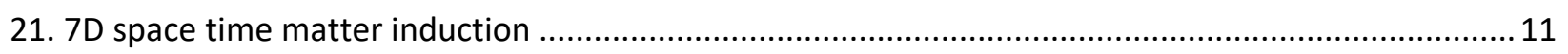

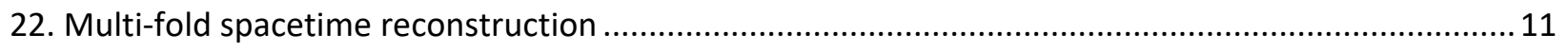

23. Spacetime reduction to 2D degrees of freedom ….................................................................... 12

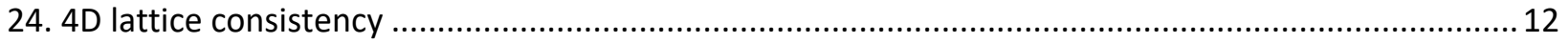

25. Higgs Bosons, Qball Condensates and Kerr-Newman Solitons ....................................................... 13

26. Multi-fold gravity electroweak symmetry breaking …................................................................. 13

27. Recovering the Standard Model and the role of Higgs field and Right-handed neutrinos.................. 14

28. Right-handed neutrinos and traversable wormholes: linking ER=EPR and Multi-folds .....................14

29. Ultimate Unification and Weak Gravity Conjecture .................................................................... 15

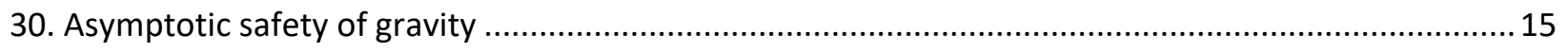

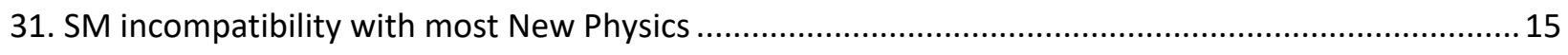

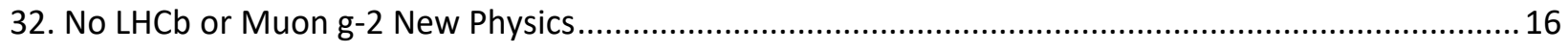

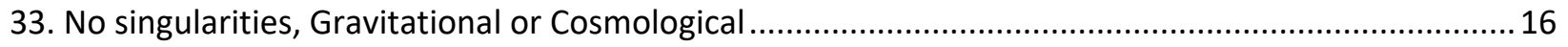

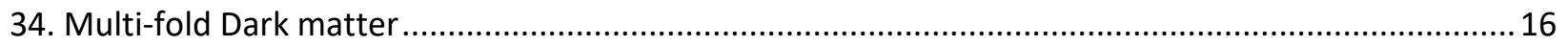

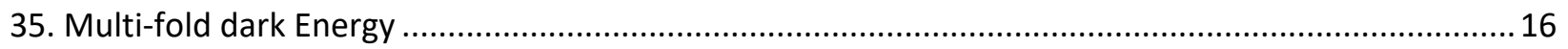

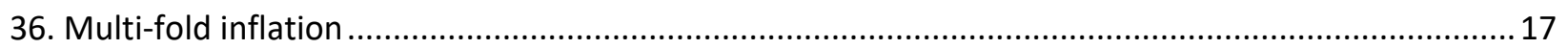

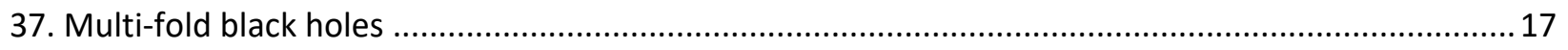

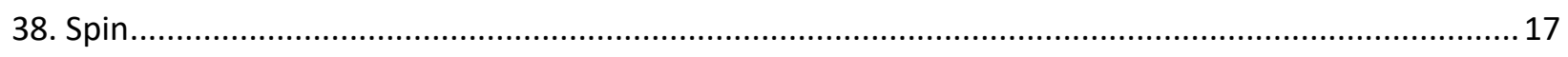

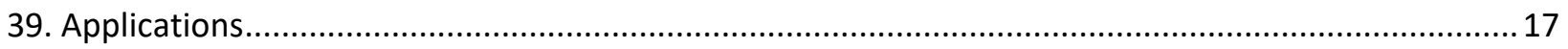




\section{Introduction}

This paper provides a synopsis of the multi-fold theory as of the date of publication, putting together the original paper [1] and the follow-up development into one consistent theory, as complete as it currently is. Feedback is welcome and encouraged. As such we also apologize for its early nature.

It is a draft and skeleton for more developed review papers in preparation for a detailed lecture or book. Feedback is therefore welcome.

The overview presented in this paper and the related research presents a new qualitative approach off the beaten path to address quantum gravity and the understanding of quantum gravity. Aspects are not fully developed, in particular a quantitative model. We do hope that it helps address some of the challenged encountered today in Physics.

Although many aspects seem to relate to our real universe, and the self consistency that we have encountered is compelling, we do not have yet any sign of confirmation. Therefore, it is presented as a model of multi-fold universes, that may or may not relate to the real universe. Physics is assumed the same up to the insights or variations introduced by our principles and model.

\section{Overview of Multi-fold theory}

In a multi-fold universe, gravity emerges from Entanglement through the multi-fold mechanisms. As a result, gravity-like effects appear in between entangled particles, that they be real or virtual. Long range, massless gravity results from entanglement of massless virtual particles. Entanglement of massive virtual particles leads to massive gravity contributions at very smalls scales. Multi-folds mechanisms also result into a spacetime that is discrete, with a random walk fractal structure and non-commutative geometry that is Lorentz invariant and where spacetime nodes and particles can be modeled with microscopic black holes. All these recover General Relativity (GR) at large scales and semi-classical models remain valid till smaller scale than usually expected. Gravity can therefore be added to the Standard Model resulting into what we defined as SMG. This can contribute to resolving several open issues with the Standard Model without New Physics other than gravity, i.e. no new particles or forces. These considerations hints at a even stronger relationship between gravity and the Standard Model.

Among the multi-fold SMG discoveries, the apparition of an-always in-flight, and hence non-interacting, righthanded neutrinos, coupled to the Higgs boson is quite notable. It is supposedly always around right-handed neutrinos, due to chirality flips by gravity of the massless Weyl fermions, induced by 7D space time matter models and hidden behind the Higgs boson or field at the entry points and exit points of the multi-folds. Massless Higgs bosons modeled as minimal microscopic black holes mark concretized spacetime location. They can condensate into Dirac Kerr-Newman soliton Qballs to produce massive and charged particles, thereby providing a microscopic explanation for a Higgs driven inflation, the electroweak symmetry breaking, the Higgs mechanism, the mass acquisition and the chirality of fermions and spacetime; all resulting from the multi-fold gravity electroweak symmetry breaking.

The multi-fold paper [1] proposes contributions to several open problems in physics, like the reconciliation of General Relativity (GR) with Quantum Physics, explaining the origin of gravity proposed as emerging from quantum (EPR- Einstein Podolsky Rosen) entanglement between particles, detailing contributions to dark matter and dark 
energy, and explaining other Standard Model mysteries without requiring New Physics beyond the Standard Model other than the addition of gravity to the Standard Model Lagrangian. All this is achieved in a multi-fold universe that may well model our real universe, which remains to be validated.

With the proposed model of [1], spacetime and Physics are modeled from Planck scales to quantum and macroscopic scales and semi-classical approaches appear valid till very small scales. In [1], it is argued that spacetime is discrete, with a random walk-based fractal structure, fractional and noncommutative at, and above Planck scales (with a 2-D behavior and Lorentz invariance preserved by random walks till the early moments of the universe). Spacetime results from past random walks of particles. Spacetime locations and particles can be modeled as microscopic black holes (Schwarzschild for photons and concretized spacetime coordinates, and metrics between Reisner Nordstrom [2] and Kerr Newman [3] for massive and possibly charged particles - the latter being possibly extremal). Although possibly surprising, [1] recovers results consistent with others (see [4] and its references), while also being able to justify the initial assumptions of black holes from the gravity or entanglement model in a multi-fold universe. The resulting gravity model recovers General Relativity at larger scale, as a 4D process, with massless gravity, but also with massive gravity components at very small scale that make gravity non-negligible at these scales. Semi-classical models also turn out to work well till way smaller scales that usually expected. [8] may also justify different coupling values at very small scales, in the 2D massless random walk regime.

All the outcomes of the multi-fold theory, so far, are compiled in $[9,10]$. This includes also our analysis and interpretations of the impact of multi-fold theory on New Physics.

\section{Multi-fold mechanisms}

The fundamental motivation for the initial model was to find a way to restore locality and common sense in (EPR) entanglement that seems to imply non-locality. To the effect, we proposed that when local entanglement takes place between two particles, multi-folds are activated, in ways that allow paths in path integrals associated to the particles to also traverse the multi-folds [1]. The entanglement is the entry point for such path. Exit points are the result of mapping of end points in the multi-fold to the evolving positions of each particles, and coincide in the multi-folds for both particles. This way it is plausible to explain how the two EPR entangled particles can at any time apparently instantaneously communicate/interact. As such, it is analogous with the idea of the ER=EPR conjecture $[5,6]$, where (entangled) black holes are connected. Yet they are typically not traversable and so do not allow paths of the path integral to traverse the connected black holes. Another model, of the same vain, suggests that locations where entangled particles are systems are connected, outside spacetime, by a minimum length segment [7]. The proposed model accounts for the Bell inequalities and non-locality with local models.

To account for exit points of the paths, when entanglement takes place, we introduced the notion of mappings from a mapping support domain in spacetime, between the entangled systems, to the multi-folds. As a result for smoothness and continuity/analyticity, any particle or system crossing the spacetime support domain of the mapping has paths of its path integral feeling the multi-folds.

Multi-folds live in a AdS(5) universe dual /tangent that surround every spacetime location. Multi-folds kinematics and dynamics follow the motions of the entangled system starting from entanglement till disentanglement, e.g. due to measurement or disturbance of the system. Deactivation detaches the mapping and shrinks the multi-folds towards the center of mass with a velocity of $c$ and so does its effect on the mapping support domain. It ensures that the theory is unitary and conserves what is conventionally conserved. That is in addition to the fact that the probability $\varepsilon$ of having a path on the multi-folds. The multi-fold dynamics a priori does not have to obey GR, although it might. 
[1] also introduces a hierarchical entanglement principle that restricts multi-fold activations to cases, where entanglement occurred locally².

A universe following these mechanisms, and where the absence of any supra luminosity, in vacuum, is enforced is called a multi-fold universe. It is assume that Physics in a multi-fold universe and in multi-folds is the same as our real universe, except for the extra multi-folds, absolutely no-supra luminosity, and the impact of that principle on path integrals, and the consequences of multi-folds theory (typically at very small scale), as well as the notions of particles and entanglements as discussed in upcoming sections.

[1] introduces a multi-fold tenancy model: one multi-fold instance per particle that "feels it" so that no interactions takes place in multi-folds other than at entry and exit points and with some exceptions ${ }^{3}$. A key motivation being that such interactions would open the door to effect not encountered so far: creation or annihilation of particles in the multi-fold or seemingly non-local interactions for gauge interactions, expected to be local ${ }^{4}$.

\section{Gravity-like fluctuations from entanglement}

The impact of paths on the multi-folds is equivalent to a potential in $\frac{1}{r}$, between the entangled particles, where $r$ is the distance from the center of mass of the entangled particles [1]. The potential is attractive towards that center of mass.

It provide a path for validation or falsifiability of key aspects of the multi-fold theory: it predicts that gravity-like fluctuations appear among entangled systems [1,12].

\section{W-type multi-fold hypothesis}

It is an additional hypothesis added later [11], that assumes that every spacetime location within the domain of a wavefunction, or field, is connected to any other location within the domain of a wavefunction or field, via multifolds that deactivate as for regular multi-fold when a wave function collapses. It offers a new interpretation of Quantum Physics and sheds new light on many aspects like BEC, the Born rule, path integrals and the measurement problem.

The W-type multi-fold hypothesis, if true, also presents the advantage to easily motivate the multi-fold mappings: overlapping support domain of overlapping wave functions will be connected this way and as a results path of a particle crossing in between entangled particles will feel the associated multi-folds as in such case we can assume a non-zero $\varepsilon$ wave function norm everywhere in between the entangled particles.

\section{Time and Time Symmetry violation}

\footnotetext{
${ }^{2}$ In $[7,8]$, we found some implications of this principles, cast in terms of the ER=EPR conjecture, where it is also essential to consistency of the model and entanglement.

${ }^{3}$ Aspects of these exceptions are discussed later on and include the presence of Higgs fields and Higgs bosons in multi-fold (motivated by the need have massive particles remaining massive on their path on the multi-folds they encounter) and right-handed neutrinos. Keep an eye on this later.

${ }^{4}$ These behavior can be kept in mind if such effects wherever to be encountered in the real universe. We introduce the tenancy principle because none of this is observed, and the tenancy principle accounts for this. We admit that tenancy principle may not apply.
} 
As a result of the multi-fold mechanisms, especially at deactivation, entanglement, especially disentanglement breaks time symmetry. It is irreversible and provide a direction for the arrow of time $[1,13,14]$.

The W-type multi-fold hypothesis extends such irreversibility to wave function collapses.

As, per $[1,15,16]$, gravity results from entanglement, gravity therefore breaks T symmetry, and it therefore probably also breaks CP symmetry if torsion is involved, which multifold mechanism support to be generated within matter (i.e. any energy particles boson or fermion in our usage of the term); otherwise, CPT is violated by gravity 5 .

As we will review in an upcoming section, time fluctuations generate space and fields of particles. Time clicks marshals the random walks of particles that construct spacetime and results from them. As particles are created in entangled pairs, time results from the random walks of entangled particles and it is entanglement that makes time observable within the system.

Time itself originates from the random walks, as the click of position changes $[1,13]$. It is also consistent with a view that time perceptions result from entanglement and can only be perceived within the universe (not as a super observer of the universe) [13].

\section{Gravity emergence from entanglement and the E/G Conjecture}

[1] proposes that any entity or energy source emits an amount of entangled pairs pf virtual particles and their antiparticles. The amount of each type being proportional to the energy or mass of the source. As a result, and assuming that multi-folds appear and behave the same way for real and virtual entangled particles, we recover an effective potential generated by each source towards it that is in $\frac{1}{r}$. A particle encountering the domain support of the multi-fold mappings, has paths of its path integral on the multi-folds and therefore feels this effect proportionally to its mass. We recover gravity. In fact, when computing this over the whole multi-fold spacetime for the distributions of mass and energy, we recover the Hilbert Einstein action and Einstein GR field equations, when considering only massless contributions.

[19] further showed that the proposed derivation of the effective potential associated to multi-fold implies recovery of the (weak) equivalent principle.

These result lead to our E/G conjecture: in a multi-fold universe, entanglement is gravity and gravity is entanglement. In other words, entanglement creates gravity effects and gravity results from entanglement effects. The conjecture is that this statement applies also to our real universe [16].

Also, in [1] and in the web version of [31] $]^{6}$, we note that conventional Physics also predicts that entanglement implies GR.

[1] also illustrates the possibility, but not implication, that multi-fold mechanisms would generate torsion, non propagating and limited to within/around matter, i.e. any boson or fermion. The presence or absence or torsion would play a role in the respect of $\mathrm{C}, \mathrm{P}$ or $\mathrm{CP}$ symmetries of gravity and entanglement.

\footnotetext{
${ }^{5}$ Of course CPT could also be violated even if CP is violated: the effective Lagrangian derived from "changes of variable" in [1] may simply not follow the forms assumed to derive CPT symmetry in QFT.

${ }^{6} \mathrm{~A} v 2$ will appear in the future. The web address contains the v2.
} 


\section{Massive and massless gravity}

All the different types of particles a contribute to the flow of entangled virtual pairs. Massless contribute till infinite ranges (unless if confined). Quasi-massless, i.e. think of neutrinos, are limited to a slightly shorter range. More and more massive particles contribute as virtual pairs at shorter ranges. As a result, multi-fold universes have massless and massive gravity contributions $[1,15]$, all stable and asymptotically safe, something we will discuss later $[17,18]$.

\section{Gravitons}

The massive and massless particles associated to entanglement and gravity can be considered as gravitons [1]. They can be seen as pairs of spacetime effective potential fluctuations that evolve as entangled particles evolve, as quasi particles $[1,20,21]$. Or they can be seen as the multi-folds in $\mathrm{AdS}(5)$, almost as closed strings in superstring theory. We will later see that if associated to quanta of spacetime, due to its discrete nature, both multi-fold and spacetime quanta are equivalent. So multi-fold and effective potential fluctuations or spacetime quanta are different facets of each other.

This duality also explains why gravitational energy seems to not be accounted for in $G R$, despite clearly being contained in gravitational waves $[1,20,21,22]$, a problem already accounted for by many like Rosen $[23,24]$ or with the challenges with the energy momentum pseudo tensor of GR (e.g. $[22,25])$.

Also, and as somehow also hinted by Rosen, although with a different model, spacetime can be seen as a flat background flat with additional effective potentials instead of curved $[1,20,21,24]$. All the models are equivalent. But I implies that only universes with positive cosmological constant can be accounted for unless if the underlying background spacetime can be differently motivated to be negatively curved, something plausible $[18,26]$ but also a key challenge for superstrings which are considered by many as incompatible with a positive cosmological constant, and therefore a prior positive curvature [26].

Despite the absence of models of gravitons as conventionally expected, the reliance on virtual particles does not lead to problems of gravity shield: changes in the potential energy of intermediated shields convey the same effective potentials further away with or without shield [39].

The symmetry of the multi-fold mechanisms is spin-2 $\left(180^{\circ}\right)$. This, and its connection to two entangled particles, also explain the double copy of gravity duality with gravity (or gravity as squared gauge theory)[66].

\section{AdS/CFT correspondence conjecture and facts in Multi-fold universes}

From the mechanisms of multi-fold universes and AdS(5) surrounding any particle or source of energy that emits pairs of entangled virtual particles, we recover a version of an holographic principle and what is conventionally known as the AdS/CFT correspondence conjecture [30] (We also reviewed its derivation in [7]), which is typically considered as a global approach à la M-theory ${ }^{7}$.

In a multi-fold universe, the correspondence is factual, and essentially conveys the duality discussed in section 9, [1]: gravity effects as effective potentials in the multi-fold spacetime result from multi-fold living in AdS(5). There

\footnotetext{
${ }^{7}$ These require $\operatorname{AdS}(5) \times S^{5}$.
} 
are however some differences: spacetime is not supersymmetric, fields are not conformant except at very high energy, i.e. at very small scales, and gravity exists in spacetime, i.e. the boundary of $\operatorname{AdS}(5)$, as well as in the bulk of $\operatorname{AdS}(5)$, as the multi-folds are present there ${ }^{8}$.

Because of the structure of entanglement, and its spin-2 symmetry, [1,31] encounters several area laws for entropy of systems separated by a suitable surface, e.g. horizon or causal horizon; which define area laws for black holes and spacetime causal horizons.

We also establish a relationship between the entropy of a region bounded by a surface in spacetime and its RyuTakayanagi area in AdS(5): in [31], it is shown to directly relate to the extent of the largest relevant multi-folds between entangled regions bounded by the surface.

\section{Particles}

Because of the multi-fold models, it is essential to be able to track particles and their entanglements [1], something that is in fact not incompatible with QFT if done for particles in between their creation and annihilation. These aspects would require additional considerations in QFT as well as in superstrings or Loop Quantum gravity $[27,28]$ and other reconstruction models [29].

\section{2. $\mathrm{SM}_{\mathrm{G}}$ Basics}

With the effective potential in $\frac{1}{r}$, the effected is stronger at small scales. This is compounded with the effects of massive gravity due to massive, short ranges interactions. $\mathrm{SM}_{\mathrm{G}}$ proposes that gravity is no more negligible at the Standard Model (SM) lengths or below [1].

$[9,10]$ compiles a whole set of effects that may be explained by $\mathrm{SM}_{\mathrm{G}}$. They are listed in the next few sections before moving on to other aspects of multi-fold universes.

Much of the SMG implications are not limited to multi-fold universes but could apply to any models where gravity is not negligible at SM scales.

On the other hand, as proposed in [1], semiclassical arguments, considered valid at $\mathrm{SM}_{\mathrm{G}}$, as in a multi-fold universe effects with the effective potential in $\frac{1}{r}$, applies to the SM scales. Variations only happen at smaller scales or higher energies, around the electroweak symmetry breaking scale ${ }^{9}$ as will be discussed later on.

\section{Gravity-induced helicity and chirality flips}

\footnotetext{
${ }^{8}$ We encountered additional justifications for gravity to possibly reign in AdS(5): [32] introduces such effects to induce space time and matter from higher dimensions (see an upcoming section), [7] sees a hint of it from the derivation of strings and gravity / GR in AdS(5) resulting from the strict AdS/CFT correspondence conjecture and $[7,32]$ see a different hint that multi-fold dynamics may follow GR in $\mathrm{AdS}(5)$ by relying on traversable wormholes for the ER-EPR conjecture where the traversability result from the derivation massive right handed neutrinos in the multi-folds (to be discussed in a later section also).

${ }^{9}$ Conventional electroweak symmetry breaking will be modeled as part of the multi-fold gravity electroweak symmetry breaking and Ultimate Unification (UU) [1,34,35].
} 
Massless particles incur helicity, and therefore chirality flips in gravity fields [1]. Stronger gravity effects increase the effect. We will see later on, that at very small scales or in the early stages of the universe, all particles are massless.

\section{Absence of proton decay}

The gravity induced chirality flips of massless fermions, including the quarks smear the anomalies around the baryon and lepton number symmetries. As a result, $[1,33]$ proposes that these symmetries must be taken much more seriously that usually expected. It has consequences that proton decay is forbidden, except maybe in black holes or if other more fundamental symmetries are broken.

The prediction of absence of proton decay and its explanation in the context of $\mathrm{SM}_{\mathrm{G}}$ is a challenge to all the theories that predicts proton decay, i.e. supersymmetry, superstrings and many Grand Unification Theories (GUTs) and Theories of Everything (TOEs). Even if it could be seen as a approximate symmetry of these theories, the argument does not works too well anymore once they claim to themselves model gravity and gravity would dominate before (i.e. lower scales or earlier age of the universe) the reign of these theories.

This results from $\mathrm{SM}_{\mathrm{G}}$ does not require the universe to be multi-fold.

\section{Absence of magnetic monopoles}

Gravity precludes magnetic monopoles. It can be seen two different ways:

- Gravity geodesics break the symmetry of Maxwell equations with sources: charges appear as current, due to the movements along the geodesics, and the symmetry disappears between electric and magnetic fields. This is a semiclassical argument, considered valid at $\mathrm{SM}_{\mathrm{G}}$, as explained in section 12 .

- Polarity and helicity/chirality flips due to gravity amount to broken symmetry between electric and magnetic fields. The model is again semi-classical

The prediction of absence of magnetic monopoles does not bode well for theories that predict them. These are uber symmetry approaches for GUTs as well as many TOEs, supersymmetry and superstrings. Again,

This result depends only on $\mathrm{SM}_{\mathrm{G}}$ and validity of semi-classical models for gravity. It does not require multi-fold universes. Even if it could be seen as an approximate symmetry of these theories, the argument does not works too well anymore once they claim to themselves model gravity and gravity would dominate before (i.e. lower scales or earlier age of the universe) the reign of these theories.

\section{The strong CP violation problem}

$[1,36]$ showed that by adding a gravity contribution to the SM Lagrangian, the up-quark mass could be reduced enough to eliminate string $\mathrm{CP}$ violation problem.

This result depends only on $\mathrm{SM}_{\mathrm{G}}$. It does not require multi-fold universes.

\section{3 is the number of fermion generations}


By adding a gravity contribution to the SM Lagrangian, one can see that only 3 regimes of Lagrangian will occur, therefore defining only three distinguishable fermions generations per family [1,37]. Such a motivation for 3 and only 3 generation of Fermions is so far considered as a mystery.

This result depends only on $\mathrm{SM}_{\mathrm{G}}$. It does not require multi-fold universes.

\section{Stabilization of the electroweak vacuum}

The idea that the electroweak vacuum may be a false vacuum has been an area of concerns for some. Since the discovery of the Higgs boson, it has been shown that nature seems to be stable but at the edge of instability.

In $[1,38]$, we show how, again, by adding a gravity contribution to the SM Lagrangian, the stability of the electroweak vacuum is reinforced by bringing it in the regime where not only it is stabler but it is also a true vacuum.

\section{Right-handed neutrinos and neutrino masses}

Adding gravity to the Lagrangian of the $\mathrm{SM}$ (i.e. $\mathrm{SM}_{\mathrm{G}}$ ) implies that left-handed neutrinos, and right-handed anti neutrinos, at very small scale (at energies electroweak symmetry breaking) can be flipped to right-handed neutrinos and left-handed anti-neutrinos, respectively $[1,7]$. Therefore, they should be present in a multi-fold universe, and therefore explain the mass of neutrinos as the result of the same mass acquisition as any other fermions.

As right-handed neutrinos have never been observed, the proposal in $[1,7]$ is that these right-handed neutrinos only exist in flight. What that means in in fact derived later on. However, at this stage of the overview of multi-fold theory, we could guess that that may mean that right-handed neutrinos, and left-handed anti neutrinos, only exist where interactions are not possible. That could within the multi-folds ${ }^{10}$.

If most of the time in flight, one can also expect that the interactions may occur less often; hence the small masses: they can only take place when chirality flip, i.e. way less often than all the other massive particles.

The result is important as it implies that neutrinos are not Majorana particles and that no high mass right-handed neutrino and other sterile neutrino exist either [40]; something reinforced by numerous particles physics experimental results as well as by section 17 .

\section{Matter antimatter asymmetry and all falling down}

The multi-fold mechanisms that generate gravity do not depend on matter or anti-matter as they result from entanglement. As a result, [41] predicts that matter and antimatter are similarly attracted, not repulsed.

With the time symmetry breaking described in section 6 , matter and antimatter interacts differently with gravity and therefore, the chirality flips occur at different rates. As the right-handed neutrino interacts less, per section 19, [41]shows that these interactions result into a deficit of antimatter.

\footnotetext{
${ }^{10}$ In upcoming sections we will see that it is indeed the case and that The Higgs boson also plays a special role.
} 


\section{7D space time matter induction}

A 4D spacetime with multi-folds is embedded in a 7D spacetime (subject to 7D GR, flat por Einsteinian). It is analogous to a unconstrained Kaluza-Klein (KK) model, i.e. KK without compactified dimensions [17]. Objects in a 4D multi-fold spacetime do not feel the embedding universe, beyond though fluctuations when entering or existing the multi-folds.

In such cases, particles, interactions and charges can be derived as the result of 7D geometrical objects. Examples are discussed in [42]. 7D spacetime is the minimum required to support the symmetries of the standard model $[43]^{11}$. Note that to support 7D GR with multi-folds would require 3 more dimensions (embedding), i.e. a 10D embedding universe with gravity (multi-fold) living in 11D (adding scale - as a dual/tangent universe). We recover the 10D and 11D properties of superstrings and M-theory argued in [43], without supersymmetry considerations.

There are a few addition interesting observations extracted from the 7D space time matter induction.

A 7D spacetime is incompatible with chiral fermions, however, in our model chiral fermions live in 4D. They only at best could feel 7D through $\varepsilon$ fluctuations when entering or exiting the multi-folds. It is also for these reasons that multi-folds are not seen in 4D multi-fold spacetime and do not require dimension compactification for the 7D embedding spacetime or the dual/tangent $\mathrm{AdS}(5)$. Therefore there is no issue having 4D chiral fermions once fermions emerge if spacetime is chiral. It turns that it is the case as discovered in [27] and explained in [34].

[42] relies mostly on numerology to show that SM particles masses could be predicted from space time matter induction. Interestingly, the Higgs boson mass and another neutral particle ( $\mathrm{M}^{*}$ references used by [42]) appear as if $5 \mathrm{D}$ particles ${ }^{12}$ at the edge of our 4D spacetime. It matters. From this observation, we propose that at the entry (or exit) of multi-folds, we have Higgs bosons and associated to them within the multi-fold another mystery particle of mass compatible within the limits on the tau neutrino mass ${ }^{13}$. With our proposal of sections 19 and 20 , we will take this to be representative of right-handed neutrinos (merged and mixed across flavors in multi-folds), and as a 7D massive object before electroweak symmetry breaking. More on this later.

\section{Multi-fold spacetime reconstruction}

The initial analysis of the multi-fold mechanisms provides hints that spacetime is actually discrete and noncommutative [1]. The main arguments being:

- No supra luminosity argues for a finite length

- $\quad \mathrm{GR}$ is recovered from a graph of microscopic black holes

- The relationship of the position in multi-fold depends on the momentum in the corresponding direction and therefore appears in AdS(5) or the 7D embedding universe. As a result, spacetime should also be non-commutative, per the mappings. Non-commutativity of spacetime also hints for finite length and discreteness [46].

\footnotetext{
${ }^{11}$ Note that the situation is different from conventional KK: because dimensions are compactified, it requires 11D [44]. Because the dimensions are not compactified, the 4D spacetime can contribute to the required dimensions (i.e. they can be used for universe that generates the gauge interactions), while when the dimensions are compact dimensions, one needs at least 7 such compactified dimensions: induction is for embedding an embedded 4D manifolds, not a product of manifolds [45].

12 Because of the symmetries in multi-fold mechanisms, for any entangled pair the process is really dominated by a 5D embedding. So 5D or \&D does not really matter for these analysis: the outcome is equivalent.

${ }^{13}$ And therefore relatable to every neutrino through mass mixing.
} 
- Quantization of gravity implies discrete spacetime

- Discretization of spacetime implies non-commutativity

- Non-commutativity provides a way to ensure Lorentz invariance

- Space and time appears to be created by random fluctuations.

- (Relativistic) Path integrals appear as fractal walks.

- Random Poisson ${ }^{14}$ distributions on a 4D spacetime lattice is (the only way to be) Lorentz invariant

In addition, particles appear in a multi-fold universe as surrounded by a microscopic black hole. It is how AdS(5) appears as dual or tangent to every spacetime point.

We therefore proposed that a multi-fold spacetime is a graph with microscopic minimum black holes at its nodes. Particles are also microscopic black holes, defined by the characteristics of the particle, more on this soon. Multifold mechanisms exist in a discretized version (in spacetime and in AdS(5)).

Path integrals result from random walk of the particles on the spacetime. Therefore, spacetime is constructed by random walk and result into a fractal structure. We propose that spacetime location that have been occupied by a random walk are concretized spacetime. It is by opposition to the other spacetime location not yet concretized. The former locations are occupied by microscopic black holes, remnants of the concretization. Doing so guarantees recovery of GR at larger scales.

A Poisson random walk ensures Lorentz invariance. It is ensured by assuming that both in the early universe or at very smalls scales, the number of concretized locations depends only on the volume (except maybe at the universes edge). We will discuss options for the early universe in an later section.

Based on the reasoning of section 22, [1] shows how particles can be associated to microscopic black holes, typically beyond extremal for charged or spinning massive particles.

\section{Spacetime reduction to 2D degrees of freedom}

Random walks reduce to a 2D process with a transition from 4D to 2D via 3D [1,46-48]; a well-known result of quantum gravity [48] and key to the good behavior of gravity (e.g. asymptotic safety, renormalizability etc.), as we will review in upcoming sections.

It is worth noting that the W-type multi-fold hypothesis [11] can be understood as the way that a particles would randomly occupy one path or another at a given moment.

The resulting spacetime and 2D gravity is in fact expected to rather be the result of a KK dimension reduction to 2 $D$ (by compactifying the other two) [8]: so multi-fold mechanisms associated to entanglement and 2D remain valid even if the gravity coupling may be modified in such a regime.

\section{4D lattice consistency}

Compatibility of a spacetime lattice, regular or fractal, with SM requires an equal number of right-handed and lefthanded chiral fermions, which cancels chiral and gravity anomalies [49]. The proposal of section 19 for the righthanded neutrinos ensures that this condition is satisfied. Doing so reinforces also the anomaly cancelation as used

\footnotetext{
${ }^{14}$ Details are added on top of [1] in [46].
} 
in section 14; with as, a result a stronger weight to be given to the symmetries associated to lepton and baryon numbers.

As a consequence, the Yang-mills mass gap is proven in a multi-fold universe $[1,50]^{15}$, thereby guaranteeing consistency and good behavior of Yang-Mills fields and therefore of the SM.

\section{Higgs Bosons, Qball Condensates and Kerr-Newman Solitons}

In [1], the highest energy, i.e. smallest scales, or earliest moment of the universe, involve spacetime construction using random walk of particles that must be massless. The particle types are not defined and may involve multiple types. However based on the analysis of section 21, it became clear that a massless Higgs boson, present at every concretized spacetime location, could be the involved particle. As we will discuss later one, analysis of the possibility to model inflation using the Higgs potential and field coupled to gravity reinforces that option [51]. It will be revisited in an upcoming section.

The theory is therefore that at very smalls scales every concretized spacetime location is occupied by a massless Higgs boson. Microscopic blackholes associated to particles can be modeled as Qballs ${ }^{16}$ with edges that match solitons, solutions of the Kerr-Newman metric with a Higgs potential solution that confines Higgs boson within the Qball; unaffecting the Higgs potential outside. Properties of the Qballs result from 7D geometry with charges defined by the associated symmetries [4]. The model matches and recovers scattering and multi-poles models of the particles as well as for example the hyperfine structure constant as a measure of the geometry of the electron Qball. Interestingly the Qball edge can be seen as a superconducting region that matches the soliton edges and regularize the singularities otherwise visible of the Kerr Newman, or Reissner Nordstrom solutions, thereby resolving the conundrum typically posed with (beyond) extremal black holes when attempting to use them to model particles.

\section{Multi-fold gravity electroweak symmetry breaking}

Section 27 provides in a microscopic description of the main part of the electroweak symmetry breaking whereby we understand how the condensation of the massless Higgs bosons amount to a symmetry breaking where the Higgs boson acquires mass and provides mass to the particles into which it condensates.

The process is accompanied by spacetime acquiring chirality, something hinted in [32], and clarified in [4]: when particle become massive at symmetry breaking or most probably during earlier fluctuations, the black hole rotation result into (local) chirality of spacetime. Any matter antimatter asymmetry will amount to selecting such a chirality.

These steps match a symmetry breaking of $\mathrm{SL}(2, \mathbb{C})$ is the symmetry group for complexified Lorentz transformations on a 4D spacetime, and it can be broken into the subgroup $\mathrm{SU}_{\mathrm{R}}(2)$ (which will be associated to the weak, part of the Standard Model, when multiplied by SU(3) for the strong interaction and U(1) for the electromagnetism) and the subgroup $\mathrm{SU}_{\mathrm{L}}(2)$ (which is associated to gravity). This ability of $\mathrm{SL}(2, \mathbb{C})$ to represent gravity is unique to 4D spacetime and relies on the decomposition of spin connections into self-dual and anti-selfdual parts. When formalized in field theory, the approach recovers the electroweak symmetry breaking and an isospin doublet that consists of the Higgs boson and a massive right-handed neutrino.

15 [49] ensures In fact that the proposed resolution is even stronger that stated in $[1,50]$.

${ }^{16}$ Such particle models treat Higgs boson confinement a lot like QCD models hadrons. 
It confirms also the model that the right-handed neutrino is associated to the Higgs boson and limited to the entry and exit points of the multi-folds.

With the chirality of spacetime, we also explain how non chiral 7D fermions will decompose into chiral fermions.

\section{Recovering the Standard Model and the role of Higgs field and Right- handed neutrinos}

[46] elaborates on the previous results and shows that:

- Non-commutativity and Group Field Theory (GFT), a global non perturbative theory of spacetime construction, derives spacetime and matter dynamics from the Higgs field (at a transition from 2D to 3D).

- Non-commutativity theory recovers bottoms-up the fermions and bosons of SM, along with the righthanded neutrino and neutrino mass mixing.

It is a remarkable corroboration of the multi-folds results obtained so far. The relationship between multi-fold theory with its concept of Higgs bosons and GFT also explains the similarities and differences with spacetime construction methods [1] especially Loop Quantum Gravity (LQG) [28]. The absence of multi-folds in LQG is related to the limitations of these theories in terms of modeling matter/particles and entanglements just as is the case for QFT or even superstrings as discussed in [1].

\section{Right-handed neutrinos and traversable wormholes: linking $E R=E P R$ and Multi-folds}

With the progress on the multi-fold theory report so far in this paper, we are ready to address the ER=EPR conjecture [52].

Analysis of the conjecture $[1,7,27]$ shows that there are many parallelism between the ER=EPR correspondence conjecture and multi-fold mechanisms: it connects two entangled black holes as an EPR bridge. In a multi-fold universe, entangled black holes should includes particles. Works derived from ER=EPR include proposals of a wormhole on the world sheet linking two entangled particles in ADS(5) [53], or entangled entropy modeling that match entanglement entropy when, and only when, the hierarchical principle mentioned in section would be satisfied.

However, no such model identifies the ER=EPR wormholes as the source of gravity. We understand it as being because no typical wormhole is traversable ${ }^{17}$ and therefore path of the path integrals are not considered to enter the wormholes. Among the recent work on traversable wormholes, a recent paper shows that traversable wormholes could exist if they contain entangled massive fermions in near their throat [8]. Per all the sections on the right-handed neutrinos, this is exactly what happens: pairs are formed at the activation of the multi-fold due to entanglements (entry point) and they stay at the level of the exit point of the multi-folds. The hierarchical entanglement principle for the multi-folds [1], and the entropy condition mentioned in [7], amount to enforcing

\footnotetext{
${ }^{17}$ They require typically to be in $\mathrm{AdS}(5)$ and exotic matter (with negative energy) although things seems to have change recently (see for example $[7,8]$ ).
} 
that the pairs of fermions at the multi-fold exit points be entangled (because initially entangled at the same entry point when the associated particle got entangled.

Mappings (at exit point) plus multi-folds formed the entangled blackholes or traversable wormholes. With this model, ER = EPR is particular case of multi-fold theory and generates gravity or at least gravity-like fluctuations in between entangled particles, thereby recovering the results of section 4, or variations of the results of section 7.

It is also worth noting that at energies above gravity electroweak symmetry breaking, spacetime fermions may not be massive. [8] address this challenge: discrete multi-folds are traversable and still good approximations of wormholes. So even in the 2D massless regime, multi-fold as worm hole would remain traversable; but the coupling constants could change.

\section{Ultimate Unification and Weak Gravity Conjecture}

$[1,35]$ detailed the ultimate unification (UU) where at high enough energy, i.e. smalls scales or in the early moments of the universe, we concluded that all interactions must become equivalent in intensity to gravity. Per section 26, the dominating particle is the massless Higgs boson. Any other particle would also only appears in pairs as fluctuations.

As a result, as discussed in [1], the weak gravity conjecture (WGC) is violated (equality instead of strict inequality) at such small scales and a new model for black hole decays and remnants can be considered.

Furthermore, sections 26 and 27 suggest no new particles above the multi-fold gravity electroweak symmetry breaking $[54]^{18}$.

\section{Asymptotic safety of gravity}

Due to the 2D random walk regime, multi-fold gravity is asymptotically safe and renormalizable as is therefore $\mathrm{SM}_{\mathrm{G}}$ $[17,18]$. In fact, we proved in [18], that this results is extensible to the real universe.

\section{SM incompatibility with most New Physics}

New Physics can be classified in two classes: new particles and new theories beyond QFT. As such we do not consider SMG or multi-fold theory to be new Physics but to rather just add gravity to SM.

The asymptotic safety of gravity implies incompatibility with theories that require more particles than what is in SM or more dimensions that say lower than 4D up to 6D. As a result most New Physics theory are not consistent:

- $\quad$ Supersymmetry because of the need of super partners

- $\quad$ Superstrings, supergravity and M-theory because of (the additional) extra dimensions ${ }^{19}$.

- Most popular GUTs and TOEs because of similar considerations

\footnotetext{
18 This will be reinforced by an upcoming section on the non-physicality of supersymmetry, superstrings and most popular GUTs and TOEs.

${ }^{19}$ Multi-fold spacetime is $4 \mathrm{D}$ and with the same number of particles as SM. At the differences of conventional KK or Superstrings, the \&D embedding spacetime is only felt through fluctuations when entering or exiting the multifolds. As such, it is not an extra dimensional theory.
} 
This is on top of the challenges already identified with proton decay (section 14) and magnetic monopoles (section $15)$, or the problem that positive cosmological constant universes seem to be part of the string swampland $[1,26]$. There is an exception though: as most gravity theory agree on the existence of a 2D regime [48], it is possible that superstrings could model correctly spacetime during that regime $[18,48]$. Unfortunately that suitability would apply only during that regime $[1,7,17,27,58]$. Also, the challenge with the string swampland is possibly resolvable in the sense that during that 2D massless result and at such very smalls scale, entanglement between freshly concretized (or encountered) spacetime location may add an additional attractive contribution that renders the cosmological constant negative at these scales $[18,26]$.

\section{No LHCb or Muon g-2 New Physics}

Along these lines and based on these conclusions, we can find plausible explanations to the hints of New Physics recently reported:

- [55] explains the problems with lepton universality with the structure of the Higgs Qballs.

- [56] suggest other reasons for potential discrepancies of the muon g-2 experimentation: the non-point like structure of the muons, coupled with its higher mass than the electron may lean itself to a Dirac Landé g-factor for the muon different from 2, instead of issues of radiative corrections ${ }^{20}$.

\section{No singularities, Gravitational or Cosmological}

In a multi-fold universe, there are no gravitational or cosmological singularities [1]. They are simply forbidden by multiple mechanisms that counter them:

- Discrete spacetime and minimal length ensure no singularity.

- Non-commutativity prevents singularities $[1,46]$.

- Torsion ensures absence of gravitational or cosmological singularities

- Multi-fold dark energy (see later) also combats attraction.

\section{Multi-fold Dark matter}

In $[1,12]$, we propose that, in multi-fold universes, dark matter effects result from the gravity-like effects associated to entanglement between real particles emitted by objects in a galaxy (attractive towards the center of mass of the real particles).

In fact Dark matter is the most obvious example of the gravity fluctuations that appear among entangled systems. [59-61] further illustrates how this proposal can handled the different situations encountered so far when it comes to dark matter.

\section{Multi-fold dark Energy}

\footnotetext{
${ }^{20}$ One should also note the possibility for incorrect theoretical computation of the QCD radiative corrections as explain in [2] and the comments on the page about [57].
} 
$[1,62]$ illustrates how fluctuations of entangled particles may create an effected potential pointing towards the embedding environment. It results into a persistent expansion force that is fueled by quantum fluctuations, not equal to the value of the vacuum energy, thereby also explaining the small value of the cosmological constant.

\section{Multi-fold inflation}

$[1,62]$ shows how high energy enough massless particles can exponentially grow spacetime which matches a model of inflation, at least in a universe with positive curvature.

[63] discusses possible ways that this initial energy could be explained.

[51] shows that Higgs massless particles and the Higgs potential can characterize inflation. In general the Higgs potential is a good approximation of the microscopic random walk effects. Something also confirmed by GFT as discussed in [46].

\section{Multi-fold black holes}

The reader can find in $[1,64]$ more details on multi-fold black holes, their area laws, entropy/information, properties and life cycle. Many aspects recover, or clarify results obtained in conventional Physics and in new Physics, even the quest for triangles [65].

\section{Spin}

The multi-fold theory provides also a way to interpret spin as the result of wave function spin [1].

\section{Applications}

[1] provides an early set of plausible as well as more far looking possible application of the theory.

\section{Conclusions}

The paper reviews at a very high level the complete story line of key contributions and results of the multi-fold theory. It put together all the contributions from [1] and subsequent papers $[9,10]$ into one complete overview, linking all the different pieces. It is work in progress and at a very high level. We plan to evolve the paper to add any new development but also to progressively beef up the details into a full review, lecture or even a book.

We publish it as a draft so to collect as much feedback, questions or comments as possible. [9] is the best place to look for updates as the drafts progress through new release of this paper or new papers, when the content reaches a next level.

\section{References}


[1]: Stephane H. Maes, (2020) "Quantum Gravity Emergence from Entanglement in a Multi-Fold Universe", viXra:2006.0088v1, (June 9, 2020). (See also https://shmaesphysics.wordpress.com/2021/03/01/quantum-gravityemergence-from-entanglement-in-a-multi-fold-universe/). (https://vixra.org/pdf/2006.0088v1.pdf)

[2]: Wikipedia, "Reissner-Nordström metric", https://en.wikipedia.org/wiki/Reissner\%E2\%80\%93Nordstr\%C3\%B6m metric. Retrieved on March 21, 2020.

[3]: Wikipedia, "Kerr-Newman metric", https://en.wikipedia.org/wiki/Kerr-Newman metric. Retrieved on March 21, 2020.

[4]: Stephane H Maes, (2021), "More on Multi-fold Particles as Microscopic Black Holes with Higgs Regularizing Extremality and Singularities", https://shmaesphysics.wordpress.com/2021/02/28/more-on-multi-fold-particlesas-microscopic-black-holes-with-higgs-regularizing-extremality-and-singularities/, February 25, 2021.

[5]: Maldacena, Juan and Susskind, Leonard (2013). "Cool horizons for entangled black holes". Fortsch. Phys. 61 (9): 781-811. arXiv:1306.0533.

[6]: Yan Breek, (2020), "Geometric Discrete Unified Theory Framework", https://www.researchgate.net/publication/341176942_Geometric Discrete Unified Theory Framework. Retrieved on March 8, 2021.

[7]: Stephane H Maes, (2020), "Circular Arguments in String and Superstring Theory from a Multi-fold Universe Perspective", viXra:2103.0195v1, https://shmaesphysics.wordpress.com/2020/10/11/circular-arguments-in-stringand-superstring-theory-from-a-multi-fold-universe-perspective/, October 5, 2020.

[8]: Stephane H Maes, (2021), "Right-handed Neutrinos and Traversable Wormholes: the key to entanglement, gravity and multi-folds extensions to ER=EPR?", https://shmaesphysics.wordpress.com/2021/04/03/right-handedneutrinos-and-traversable-wormholes-the-key-to-entanglement-gravity-and-multi-folds-extensions-to-erepr/, April 3, 2021.

[9]: Stephane Maes, (2020), "Web Site Tracking all Publications around the Multi-fold universe", Navigation page listing all papers. https://shmaesphysics.wordpress.com/shmaes-physics-site-navigation/.

[10]: Stephane Maes, (2021), "Current Review - All Publications around the Multi-fold universe - February 2021", https://osf.io/8b69k, https://shmaesphysics.wordpress.com/shmaes-physics-site-navigation/, February 15, 2021. (More recent updates available at the URL).

[11]: Stephane H Maes, (2020), "The W-type Multi-Fold Hypothesis and Quantum Physics Interpretation of wave Functions and QFT", https://shmaesphysics.wordpress.com/2020/12/24/the-w-type-multi-fold-hypothesis-andquantum-physics-interpretation-of-wave-functions-and-gft/, December 20, 2020.

[12]: Stephane H Maes, (2020), "Gravity-like Attractions and Fluctuations between Entangled Systems?", viXra:2010.0010v1, https://shmaesphysics.wordpress.com/2020/06/25/gravity-like-attractions-andfluctuations-between-entangled-systems/, June 24, 2020.

[13]: Stephane H Maes, (2020), "Entanglement Concretizes Time in a Multi-fold Universe", viXra:2010.0083v1, https://shmaesphysics.wordpress.com/2020/06/28/entanglement-concretizestime-in-a-multi-fold-universe/, June 28, 2020.

[14]: Stephane H Maes, (2020), "A Multi-fold Universe Genesis Inspired By Explosive Total Collision: The Source Of The Big Bang?", https://shmaesphysics.wordpress.com/2021/01/17/a-multi-fold-universe-genesis-inspired-bytotal-explosion-collision-the-source-of-the-big-bang/, January 12, 2021. 
[15]: Stephane H Maes, (2020), "Massless and Massive Multi-Gravity in a Multi-fold Universe", viXra:2010.0095v1, https://shmaesphysics.wordpress.com/2020/06/30/massless-and-massive-multigravity-in-a-multi-fold-universe/, June 19, 2020.

[16]: Stephane H Maes, (2020), "The E/G conjecture: entanglement is gravity and gravity is entanglement", viXra:2010.0139v1, https://shmaesphysics.wordpress.com/2020/10/15/the-e-g-conjectureentanglement-is-gravity-and-gravity-is-entanglement/, October 15, 2020.

[17]: Stephane H Maes, (2020), "Renormalization and Asymptotic Safety of Gravity in a Multi-Fold Universe: More Tracking of the Standard Model at the Cost of Supersymmetries, GUTs and Superstrings", viXra:2102.0137v1, https://shmaesphysics.wordpress.com/2020/09/19/renormalization-andasymptotic-safety-of-gravity-in-a-multi-fold-universe-more-tracking-of-the-standard-model-at-the-cost-ofsupersymmetries-guts-and-superstrings/, September 18, 2020.

[18]: Stephane H Maes, (2021), "Quantum Gravity Asymptotic Safety from 2D Universal Regime and Smooth Transition to Dual Superstrings", https://shmaesphysics.wordpress.com/2021/02/07/quantum-gravityasymptotic-safety-from-2d-universal-regime-and-smooth-transition-to-dual-superstrings/, January 29, 2021.

[19]: Stephane H Maes, (2020), "Derivation of the Equivalence Principle in a Multi-fold Universe", viXra:2010.0090v1, https://shmaesphysics.wordpress.com/2020/06/29/derivation-of-the-equivalenceprinciple-in-a-multi-fold-universe/, June 19, 2020.

[20]: Stephane H Maes, (2020), "Multi-fold Gravitons In-N-Out Spacetime", viXra:2010.0155v1, https://shmaesphysics.wordpress.com/2020/07/27/multi-fold-gravitons-in-n-out-spacetime/, July 27, 2020, (posted September 6, 2020).

[21]: Stephane H Maes, (2020), "Particles, Especially Virtual Particles, in a Multi-fold Universe vs. QFT", viXra:2010.0133v1, https://shmaesphysics.wordpress.com/2020/07/11/particles-especially-virtual-particlesin-a-multi-fold-universe-vs-qft/, July 10, 2020.

[22]: Michael Weiss and John Baez, (2017), "Is Energy Conserved in General Relativity?", https://math.ucr.edu/home/baez/physics/Relativity/GR/energy gr.html. Retrieved for this paper on April 25, 2021.

[23]: Rosen, Nathan (1973), “A bi-metric Theory of Gravitation”, Gen. Rel. Grav., 4 (6): 435-447

[24]: Rosen, Nathan (1940). “General Relativity and Flat Space. I”. Physical Review. 57 (2): 147-150.

[25]: Wikipedia, "Belinfante-Rosenfeld_stress-energy_tensor", https://en.wikipedia.org/wiki/Belinfante\%E2\%80\%93Rosenfeld stress\%E2\%80\%93energy tensor. Retrieved on December 12, 2019.

[26]: Stephane H Maes, (2021), "The String Swampland and de Sitter Vacua: A Consistent Perspective for Superstrings and Multi-fold Universes", https://shmaesphysics.wordpress.com/2021/01/12/the-string-swamplandand-de-sitter-vacua-a-consistent-perspective-for-superstrings-and-multi-fold-universes/, January 9, 2021.

[27]: Stephane H Maes, (2020), "Dualities or Analogies between Superstrings and Multi-fold Universe", viXra:2006.0178v1, https://shmaesphysics.wordpress.com/2020/06/14/dualities-or-analogies-betweensuperstrings-and-multi-fold-universes/, June 14, 2020.

[28]: Stephane H Maes, (2020), "Alignments and Gaps Between Multi-fold Universes And Loop Quantum Gravity", viXra:2006.0229v1, https://shmaesphysics.wordpress.com/2020/06/19/multi-fold-universes-analysis-ofloop-quantum-gravity/, June 18, 2020. 
[29]: Johannes Thuringen, (2015), "Discrete quantum geometries and their effective dimension", Ph.D. Thesis, Humboldt-Universitat zu Berlin".

[30]: J. M. Maldacena, (1998), "The Large N Limit Of Superconformal Field Theories And Supergravity", Adv. Theor. Math. Phys. 2, 231

[31]: Stephane H Maes, (2020), "Area Laws Between Multi-Fold Universes and AdS", viXra:2010.0207v1, https://shmaesphysics.wordpress.com/2020/08/10/area-laws-between-multi-folduniverses-and-ads/, August 10, 2020.

[32]: Stephane H Maes, (2020), "Tracking Down The Standard Model With Gravity In Multi-Fold Universes", viXra:2011.0208v1, https://shmaesphysics.wordpress.com/2020/08/30/tracking-down-the-standardmodel-with-gravity-in-multi-fold-universes/, August 20, 2020.

[33]: Stephane H Maes, (2020), "Gravity Induced Anomalies Smearing in Standard Model so that Protons May Never Decay, Except in Black Holes", viXra:2006.0128v1, https://shmaesphysics.wordpress.com/2020/06/12/protons-may-never-decay-except-in-black-holes/, June 13, 2020.

[34]: Stephane H Maes, (2020), "Multi-fold Gravity-Electroweak Theory and Symmetry Breaking", https://shmaesphysics.wordpress.com/2021/03/28/multi-fold-gravity-electroweak-theory-andsymmetry-breaking/, March 16, 2021.

[35]: Stephane H Maes, (2020), "Ultimate Unification: Gravity-led Democracy vs. UberSymmetries", viXra:2006.0211v1, https://shmaesphysics.wordpress.com/2020/06/16/ultimate-unification-gravityled-democracy-vs-uber-symmetries/, June 16, 2020.

[36]: Stephane H Maes, (2020), "Strong CP Violation Tamed in The Presence of Gravity", viXra:2007.0025v1, https://shmaesphysics.wordpress.com/2020/06/23/strong-cp-violation-tamed-inthe-presence-of-gravity/, June 21, 2020.

[37]: Stephane H Maes, (2020), "Gravity Dictates the Number of Fermion Generations: 3", viXra:2007.0068v1, https://shmaesphysics.wordpress.com/2020/06/24/gravity-dictates-the-number-of-fermiongenerations-3/, June 24, 2020.

[38]: Stephane H Maes, (2020), "Gravity Stabilizes Electroweak Vacuum - No Bubble of Nothing to Worry About!", viXra:2007.0173v1, https://shmaesphysics.wordpress.com/2020/06/24/gravity-stabilizes-electroweakvacuum-no-bubble-of-nothing-to-worry-about/, June 24, 2020.

[39]: Stephane H Maes, (2020), "No Gravity Shield in Multi-folds Universes", viXra:2010.0032v1, https://shmaesphysics.wordpress.com/2020/06/26/no-gravity-shields-in-multifolds-universes/, June 26, 2020.

[40]: Stephane H Maes, (2020), "No Conventional Sterile Neutrinos In a Multi-fold Universe: just SMG business as usual", viXra:2103.0202v1, https://shmaesphysics.wordpress.com/2020/10/02/no-conventional-sterile-neutrinosin-a-multi-fold-universe-just-smg-business-as-usual/, October 1, 2020.

[41]: Stephane H Maes, (2020), "More Matter Than Antimatter, All Falling Down", viXra:2010.0121v2, https://shmaesphysics.wordpress.com/2020/07/05/more-matter-than-antimatter-allfalling-down/, July 5, 2020. (V2: April 8, 2021).

[42]: Stephane H. Maes, (2020) "Particles of The Standard Model In Multi-Fold Universes", https://shmaesphysics.wordpress.com/2020/11/05/particles-of-the-standard-model-in-multi-folduniverses/, November 4, 2020. 
[43]: David Bailint and Alex Love, (1987), "Kaluza-Klein theories", Rep. Prog. Phys. 50 (1987) 1087-1170.

[44]: Sabine Hossenfelder, (2021), "Does the Universe have Higher Dimensions? Part 1", Back Reaction, https://backreaction.blogspot.com/2021/04/does-universe-have-higher-dimensions.html. Retrieved on April 10, 2021.

[45]: Stephane H Maes, (2021), "Comments on dimensions required for KK to generate the Standard Moddl intercations", https://shmaesphysics.wordpress.com/2020/08/30/tracking-down-the-standard-model-withgravity-in-multi-fold-universes/\#comment-2049.

[46]: Stephane H Maes, (2021), "Multi-fold Non-Commutative Spacetime, Higgs and The Standard Model with Gravity", https://shmaesphysics.wordpress.com/2021/04/18/multi-fold-non-commutative-spacetime-higgs-andthe-standard-model-with-gravity/, April 11, 2021.

[47]: Stephane H Maes, (2021), “"Quantum Gravity Emergence from Entanglement in a Multi-Fold Universe”: 2D or 2+1D spacetime at small scales", viXra:2103.0142, https://shmaesphysics.wordpress.com/2021/03/20/quantumgravity-emergence-from-entanglement-in-a-multi-fold-universe-2d-or-21d-spacetime-at-small-scales/, March 20, 2021.

[48]: Stephane H Maes, (2021), "Spacetime and Gravity are 2D around Planck Scales: A Universal Property of Consistent Quantum Gravity", https://shmaesphysics.wordpress.com/2021/03/23/spacetime-and-gravity-are-2daround-planck-scales-a-universal-property-of-consistent-quantum-gravity/, March 20, 2021.

[49]: Stephane H Maes, (2020), "Viable Lattice Spacetime and Absence of Quantum Gravitational Anomalies in a Multi-fold Universe", https://shmaesphysics.wordpress.com/2020/12/13/viable-lattice-spacetime-and-absenceof-quantum-gravitational-anomalies-in-a-multi-fold-universe/, December 4, 2020.

[50]: Stephane H Maes, (2020), " Progress on Proving the Mass gap for Yang Mills and Gravity (maybe it's already proved...)", viXra:2006.0155v1, https://shmaesphysics.wordpress.com/2020/06/12/progresses-on-proving-themass-gap-for-yang-mills-and-gravity-maybe-its-already-proven/, June 12, 2020.

[51]: Stephane H Maes, (2020), "Multi-fold Higgs Fields and Bosons", https://shmaesphysics.wordpress.com/2020/11/10/multi-fold-higgs-fields-and-bosons/, November 6, 2020.

[52]: Maldacena, Juan and Susskind, Leonard (2013). "Cool horizons for entangled black holes". Fortsch. Phys. 61 (9): 781-811. arXiv:1306.0533.

[53]: Julian Sonner, (2013), "Holographic Schwinger Effect and the Geometry of Entanglement", arXiv:1307.6850v3.

[54]: Stephane H Maes, (2020), "New Physics is often not so new", https://shmaesphysics.wordpress.com/2021/04/27/new-physics-is-often-not-so-new/, April 27, 2021.

[55]: Stephane H Maes, (2020), “New Physics with LHCb to explain loss of lepton universality, or just gravity?", viXra:2103.0191v1, https://shmaesphysics.wordpress.com/2021/03/29/new-physics-with-lhcb-toexplain-loss-of-lepton-universality-or-just-gravity/, March 29, 2021.

[56]: Stephane H. Maes, "A bold prediction on the muon anomalous magnetic moment, and expected results to be published on April 7, 2021 by the Fermilab Muon g-2, and its explanation", viXra:2104.0030v1, https://shmaesphysics.wordpress.com/2021/04/01/a-bold-prediction-on-themuon-anomalous-magnetic-moment-and-expected-resulted-to-be-published-on-april-7-2021-by-the-fermilabmuon-g-2-and-its-explanation/, April 1, 2021. 
[57]: Sz. Borsanyi, Z. Fodor, J. N. Guenther, C. Hoelbling, S. D. Katz, L. Lellouch, T. Lippert, K. Miura, L. Parato, K. K. Szabo, F. Stokes, B. C. Toth, Cs. Torok, L. Varnhorst, (2021), "Leading hadronic contribution to the muon magnetic moment from lattice QCD", arXiv:2002.12347v3

[58]: Stephane H Maes, (2020), "Superstrings Encounter of the Second, Third or Fourth Types?", viXra:2010.0140v1, https://shmaesphysics.wordpress.com/2020/07/19/superstrings-encounter-of-the-secondthird-or-fourth-types/, July 5, 2020.

[59]: Stephane H Maes, (2020), “Multi-Fold Universe Dark Matter Successful Explanation and the "Too Thin Universe" but "Too Strong Gravity Lensing by Galaxy Clusters"”, viXra:2102.0079v1, https://shmaesphysics.wordpress.com/2020/09/15/multi-fold-universe-dark-mattersuccessful-explanation-and-the-too-thin-universe-but-too-strong-gravity-lensing-by-galaxy-clusters/, September 14, 2020.

[60]: Stephane H Maes, (2020), "Multi-Fold Universe Dark Matter Effects Survive Low-Mass Galaxies with Dark Matter Deficits and Excesses", https://shmaesphysics.wordpress.com/2020/10/14/multi-fold-universe-darkmatter-effects-survive-low-mass-galaxies-with-dark-matter-deficits-and-excesses/, October 14, 2020.

[61]: Stephane H Maes, (2020), "Multi-Fold Dark Matter Effects and Early Supermassive Black Holes", https://shmaesphysics.wordpress.com/2020/10/15/multi-fold-dark-matter-effects-and-early-supermassive-blackholes/, October 15, 2020.

[62]: Stephane H Maes, (2020), "Explaining Dark Energy, Small Cosmological Constant and Inflation Without New Physics?", viXra:2006.0261v1, https://shmaesphysics.wordpress.com/2020/06/19/explaining-dark-energy-smallcosmological-constant-and-inflation-without-new-physics/, June 19, 2020.

[63]: Stephane H Maes, (2020), “A Multi-fold Universe Genesis Inspired By Explosive Total Collision: The Source Of The Big Bang?", https://shmaesphysics.wordpress.com/2021/01/17/a-multi-fold-universe-genesis-inspired-bytotal-explosion-collision-the-source-of-the-big-bang/, January 12, 2021.

[64]: Stephane H Maes, (2020), “Multi-Fold Black Holes: Entropy, Evolution and Quantum Extrema”, https://shmaesphysics.wordpress.com/2020/11/01/multi-fold-black-holes-entropy-evolution-andquantum-extrema/, October 31, 2020.

[65]: Stephane H. Maes, (2021), "Comments on triangles and multi-fold inflation", https://shmaesphysics.wordpress.com/2020/06/19/explaining-dark-energy-small-cosmological-constant-andinflation-without-new-physics/comment-page-1/\#comment-2183.

[66]: Stephane H. Maes, (2021), "Multi-fold explains why Gravity as the Square of Gauge Theory makes sense" https://shmaesphysics.wordpress.com/2021/02/28/more-on-multi-fold-particles-as-microscopic-black-holes-withhiggs-regularizing-extremality-and-singularities/comment-page-1/\#comment-2235. Paper is in preparation. 